\title{
Corrigendum: The Adipocyte and Adaptive Immunity
}

\author{
Jianfeng Song ${ }^{1,2}$ and Tuo Deng ${ }^{1,2,3 *}$ \\ ${ }^{1}$ National Clinical Research Center for Metabolic Diseases, and Department of Metabolism and Endocrinology, The Second \\ Xiangya Hospital of Central South University, Changsha, China, ${ }^{2}$ Key Laboratory of Diabetes Immunology, Ministry of \\ Education, and Metabolic Syndrome Research Center, The Second Xiangya Hospital of Central South University, Changsha, \\ China, ${ }^{3}$ Clinical Immunology Center, The Second Xiangya Hospital of Central South University, Changsha, China
}

Keywords: adipocyte, adaptive immunity, adipokine, T cell, B cell

\section{A Corrigendum on:}

\section{OPEN ACCESS}

Approved by: Frontiers Editorial Office, Frontiers Media SA, Switzerland

*Correspondence: Tuo Deng

dengtuo@csu.edu.cn

Specialty section:

This article was submitted to Immunological Tolerance and Regulation, a section of the journal Frontiers in Immunology

Received: 27 September 2021 Accepted: 28 September 2021

Published: 08 October 2021

Citation:

Song J and Deng T (2021) Corrigendum: The Adipocyte and Adaptive Immunity.

Front. Immunol. 12:784125. doi: 10.3389/fimmu.2021.784125
The Adipocyte and Adaptive Immunity

By Song J and Deng T (2020). Front. Immunol. 11:593058. doi: 10.3389/fimmu.2020.593058

In the published article, there was an error in Affiliation 1.

Instead of "National Clinical Research Center for Metabolic Diseases, Key Laboratory of Diabetes Immunology (Central South University), Ministry of Education, Metabolic Syndrome Research Center, and Department of Metabolism and Endocrinology, The Second Xiangya Hospital of Central South University, Changsha, China", it should be split into two separate affiliations "National Clinical Research Center for Metabolic Diseases, and Department of Metabolism and Endocrinology, The Second Xiangya Hospital of Central South University, Changsha, China" and "Key Laboratory of Diabetes Immunology, Ministry of Education, and Metabolic Syndrome Research Center, The Second Xiangya Hospital of Central South University, Changsha, China".

The authors apologize for this error and state that this does not change the scientific conclusions of the article in any way. The original article has been updated.

Publisher's Note: All claims expressed in this article are solely those of the authors and do not necessarily represent those of their affiliated organizations, or those of the publisher, the editors and the reviewers. Any product that may be evaluated in this article, or claim that may be made by its manufacturer, is not guaranteed or endorsed by the publisher.

Copyright $\odot 2021$ Song and Deng. This is an open-access article distributed under the terms of the Creative Commons Attribution License (CC BY). The use, distribution or reproduction in other forums is permitted, provided the original author(s) and the copyright owner(s) are credited and that the original publication in this journal is cited, in accordance with accepted academic practice. No use, distribution or reproduction is permitted which does not comply with these terms. 